\title{
Associations Among Coagulation-Related Variables, Resolution of Disseminated Intravascular Coagulation (DIC), and Mortality in Patients with Sepsis-Induced DIC Treated with Recombinant Human Soluble Thrombomodulin: A Retrospective Observational Study
}

Chieko Mitaka ( $\sim$ c-mitaka@juntendo.ac.jp )

Juntendo University Faculty of Medicine https://orcid.org/0000-0001-5671-1168

Izumi Kawagoe

Juntendo University - Hongo Campus: Juntendo Daigaku

Daizoh Satoh

Juntendo Daigaku - Hongo Campus: Juntendo Daigaku

Masakazu Hayashida

Juntendo Daigaku - Hongo Campus: Juntendo Daigaku

Research Article

Keywords: Disseminated intravascular coagulation, Platelet, Sepsis, Prothrombin international normalized ratio, Recombinant human soluble thrombomodulin

Posted Date: May 27th, 2021

DOI: https://doi.org/10.21203/rs.3.rs-558224/v1

License: (c) (i) This work is licensed under a Creative Commons Attribution 4.0 International License.

Read Full License 


\section{Abstract}

Background: We evaluated associations among coagulation-related variables, resolution of disseminated intravascular coagulation (DIC), and mortality in patients with sepsis-induced DIC treated with recombinant human soluble thrombomodulin (rTM).

Methods: We retrospectively investigated patients with sepsis-induced DIC treated with rTM. Changes in coagulation-related variables before and after treatment with rTM were examined. Further, associations between coagulation-related variables and DIC resolution were evaluated.

Results: A total of 123 patients were included. The platelet count, prothrombin international normalized ratio (PT-INR), and fibrin/fibrinogen degradation products (FDP) significantly $(p<0.001)$ improved after rTM administration in survivors $(n=98)$, but not in nonsurvivors $(n=25)$. However, the DIC score significantly $(p<0.001)$ reduced not only in survivors but also in nonsurvivors. PT-INR before rTM was significantly $(p=0.0029)$ lower in patients attaining than not attaining DIC resolution $(\mathrm{n}=87$ and 36 , respectively). The 28-day mortality was significantly lower in patients attaining than not attaining DIC resolution $(11.5 \%$ vs. $41.7 \%, p=0.0001)$.

Conclusions: The DIC score significantly reduced after rTM in both survivors and nonsurvivors. rTM might play an important role in improving DIC, especially when treatment with rTM is initiated in the early phase of DIC.

\section{Background}

Sepsis is the most common cause of disseminated intravascular coagulation (DIC), which leads to organ dysfunction through intravascular thrombosis $[1,2]$. Inflammation deregulates the coagulation cascade and leads to various coagulopathies including DIC in septic patients $[2,3,4]$. The endothelium plays a key role in all major pathways involved in the cross-talk between inflammation and coagulation [4]. The essential therapy in patients with sepsis is treatment of underlying infection using appropriate antibiotics and source controls. However, DIC can persist or develop even after the initiation of appropriate therapy. In such situations, supportive therapy to manage DIC and to modulate cross-talk between inflammation and coagulation may reduce morbidity and mortality in patients with sepsis [4].

Thrombomodulin (TM), which is localized in the vascular endothelium, acts as an anticoagulant. After it binds to thrombin, the thrombin-TM complex activates protein $\mathrm{C}$ to produce activated protein $\mathrm{C}$, which inactivates factors Va and VIIla, thereby inhibiting formation of thrombin (Ila) [5, 6]. Furthermore, TM attenuates inflammation through various mechanisms $[7,8]$. TM directly interferes with complement activation via the classical and lectin pathways and modulates innate immunity [9]. TM binds highmobility group-B1 DNA-binding protein (HMGB1) and suppresses inflammation [10], subsequently promoting degradation of HMGB1 [11]. Since HMGB1 is a late cytokine mediator of endotoxin lethality and systemic inflammation, TM may protect septic patients also by decreasing HMGB1 [12]. 
A phase III randomized controlled trial (RCT) comparing efficacies of recombinant human soluble TM (rTM) and heparin in patients with DIC showed that rTM improved DIC and relieved bleeding symptoms more effectively than heparin [13]. Accordingly, rTM was approved as an agent for treatment of DIC in 2008 in Japan, and has since been used clinically. A multicenter propensity score analysis of sepsisinduced DIC reported that rTM reduced in-hospital mortality in patients requiring ventilation [14]. In addition, a multicenter retrospective study comparing 452 propensity score-matched pairs of septic patients with DIC revealed that rTM significantly reduced in-hospital mortality [15]. Although the Sepsis Coagulopathy Asahi Recombinant LE Thrombomodulin (SCARLET) trial performed as a multicenter RCT found that rTM did not significantly reduce 28-day mortality compared with a placebo in patients with sepsis-associated coagulopathy [16], a post-hoc analysis of this trial revealed that the subgroup of rTMtreated patients with higher baseline levels of prothrombin fragment 1.2 and thrombin-antithrombin complex had lower mortality than placebo-treated patients [17]. Therefore, rTM may play an important role in improving coagulopathy and mortality in septic patients with DIC.

The aim of the present study was to evaluate associations among inflammation- or coagulation-related variables, resolution of DIC, and mortality in patients with sepsis-induced DIC treated with rTM.

\section{Methods}

\section{Study design and subjects}

Prior to this retrospective observational study, the study protocol was approved by the Ethics Committee of Juntendo University Hospital (approval number 19-045), with a waiver of patients' written informed consent.

We retrospectively studied patients who were diagnosed with sepsis-induced DIC and treated with rTM in the intensive care unit at Juntendo University Hospital between April, 2015 and December, 2019. Sepsis was defined according to the Sepsis-3 criteria [18]. DIC was diagnosed based on a DIC score $\geq 4$ points on the Japanese Association for Acute Medicine (JAAM) DIC score [19], which is the sum of the points (graded 0 to 3 ) for the following four components: systemic inflammatory response syndrome criteria $\geq 3$ is scored as 1; a platelet count $\geq 80$ and $<120 \times 10^{9} / \mathrm{L}$ or $\mathrm{a}>30 \%$ decrease within $24 \mathrm{~h}$ is scored as 1 , a platelet count $<80 \times 10^{9} / \mathrm{L}$ or a $>50 \%$ decrease within $24 \mathrm{~h}$ is scored as 3 ; a prothrombin international normalized ratio (PT-INR) $\geq 1.2$ is scored as 1 ; a fibrin/fibrinogen degradation products (FDP) level $\geq 10$ and $<25 \mu \mathrm{g} / \mathrm{mL}$ is scored as 1 , and a FDP level $\geq 25 \mu \mathrm{g} / \mathrm{mL}$ is scored as 3 .

rTM at a dose of $380 \mathrm{U} / \mathrm{kg}$ was intravenously infused for 30 min once a day, which was usually continued for 6 days or until improvement of DIC was achieved. In patients with severe renal dysfunction, the dose was reduced to $130 \mathrm{U} / \mathrm{kg}$, considering its elimination by the kidneys.

\section{Data collection}


Data on patients' clinical characteristics and laboratory tests data were collected from medical records, including age, sex, the outcome (28-day mortality), the Acute Physiology and Chronic Health Evaluation II (APACHE II) score [20], the Sequential Organ Failure Assessment (SOFA) score [21], the DIC score [19], the platelet count, PT-INR values, levels of FDP and C-reactive protein (CRP), surgical interventions, sites of infection, infecting microorganisms, documented bacteremia, and the dose and duration of rTM administration.

Resolution of DIC was defined as the DIC score $\leq 3$ on the day after the last treatment with rTM [22]. To assess the efficacy of rTM, coagulation- and inflammation-related variables before and after treatment with rTM were examined and compared between survivors and nonsurvivors. In addition, these variables before rTM were compared between patients attaining and not attaining DIC resolution.

\section{Statistical analysis}

Qualitative data are expressed as numbers (percentages) and were analyzed using the chi-square test. Quantitative data are expressed as medians [interquartile ranges: IQRs]. Changes in variables over time were analyzed with the Wilcoxon signed-rank test. Variables were compared between dichotomized groups with the Mann-Whitney Utest. A $p$ value less than 0.05 was considered statistically significant.

\section{Results}

\section{Patient characteristics}

A total of 123 patients were diagnosed with sepsis-induced DIC and treated with rTM. Baseline clinical characteristics in these patients are shown in Table 1. The 28-day mortality rate in the total cohort was $20.6 \%(25 / 123)$. APACHE II and SOFA scores were significantly lower in survivors than in nonsurvivors ( $p$ $<0.01)$. However, other variables, including age, sex, requirements of surgery, sites of infection, infecting microorganisms including antibiotic-resistant bacteria, or documented bacteremia, were not different between survivors and nonsurvivors.

\section{Effects of rTM on coagulation- and inflammation-related variables}

In the total cohort, the dose and duration of rTM administration were 380 [130-380] $\mathrm{U} / \mathrm{kg} / \mathrm{day}$ and 5 [36] days, respectively. These were 380 [192.5-380] $\mathrm{U} / \mathrm{kg} /$ day and 5 [3.25-6] days in survivors, and 380 $[130-380] \mathrm{U} / \mathrm{kg} /$ day and 6 [3-7] days in nonsurvivors.

Coagulation- and inflammation-related variables before and after rTM administration are shown in Table 2. Before treatment with rTM, the platelet count, FDP levels, the DIC score, or CRP levels were not significantly different between survivors and nonsurvivors, and only PT-INR was significantly lower in survivors than in nonsurvivors $(p=0.0395)$. 
After treatment with rTM, the platelet count increased significantly $(p<0.0001)$ while PT-INR values, FDP levels, CRP levels, and the DIC score decreased significantly $(p<0.0001)$ in the total cohort, and also in survivors. On the other hand, in nonsurvivors, the platelet count, PT-INR values, or FDP levels did not change significantly, although the DIC score and CRP levels decreased significantly $(p<0.001)$, albeit to lesser extents compared with survivors. After treatment with rTM, the platelet count was significantly higher while PT-INR values, FDP levels, and CRP levels, and the DIC score were significantly lower in survivors than in nonsurvivors ( $p=0.0038$ for FDP, and $p<0.0001$ for others). The DIC resolution rate was significantly higher in survivors than in nonsurvivors (77/98 [78.6\%] vs. 10/25 [40.0\%], $p<0.001)$.

Comparisons of variables before treatment with rTM between patients attaining DIC resolution $(n=87)$ and those not attaining DIC resolution $(n=36)$ are shown in Table 3. The APACHE II, SOFA, and DIC scores, and PT-INR values were significantly lower while the platelet count was significantly higher in patients attaining than not attaining DIC resolution ( $p<0.01$, respectively). Other variables, including demographic data or levels of FDP and CRP were not different between these patients. The 28-day mortality was significantly lower in patients attaining than not attaining DIC resolution (10/87 [11.5\%] vs. $15 / 36[41.7 \%], p=0.0001)$.

There were no major rTM-related significant adverse events, including bleeding, in any patients.

\section{Discussion}

In the present study, we investigated mortality and DIC resolution in patients with sepsis-induced DIC who were treated with rTM. Comparisons between survivors and nonsurvivors showed that mortality was significantly associated with the APCHE II as well as SOFA score and PT-INR values before treatment with rTM, whereas it was not associated with the DIC score, the platelet count, or levels of FDP and CRP. After treatment with rTM, the DIC score and CRP levels significantly decreased in the total cohort, and not only in survivors but also in non-survivors. However, decreases in the DIC score and CRP levels were significantly less in nonsurvivors. Further, although the platelet count, PT-INR values, and FDP levels improved significantly after treatment with rTM in the total cohort, and also in survivors, they did not improve significantly in nonsurvivors. The DIC resolution rate was significantly higher in survivors than in nonsurvivors (78.6 \% vs. $40.0 \%$ ). Comparisons between patients attaining and not attaining DIC resolution showed that DIC resolution was significantly associated with APACH II, SOFA, and DIC scores, the platelet count, and PT-INR values before treatment with rTM, but not with FDP or CRP levels. The mortality was significantly lower in patients attaining than not attaining DIC resolution $(11.5 \%$ vs. 41.7 $\%)$.

Reportedly, endothelial expressions of TM and protein $\mathrm{C}$ receptors were significantly reduced in patients with sepsis [23]. Accordingly, replacement with rTM is expected to compensate for such downregulations of the endothelial thrombomodulin-protein $\mathrm{C}$ receptor pathway in septic patients. Although the primary anticoagulant effect of rTM is mediated by activated protein $\mathrm{C}$, plasma activated protein $\mathrm{C}$ levels did not increase after administration of rTM in septic patients with DIC [24]. These results suggest that activated 
protein $\mathrm{C}$ acts only locally and does not circulate systemically in response to rTM administration. Accordingly, the risk of bleeding associated with rTM could be significantly lower, compared with heparin [13]. Indeed, we did not experience any significant adverse events related to rTM, including bleeding, in the present study.

TM, which is localized in the vascular endothelium, integrates biological pathways related to coagulation, innate immunity, and inflammation, and TM thus has anti-inflammatory as well as anticoagulation effects $[5-8,10]$. In particular, the N-terminal lectin-like domain of rTM attenuates inflammation through inhibition of leukocyte adhesion to endothelial cells, inhibition of complement pathways, and degradation of HMGB $1[8,9]$. The recombinant lectin-like domain of rTM binds to Gram-negative bacteria and lipopolysaccharides, enhances their elimination from the body, and protects the host by suppressing inflammatory responses [25]. In the present study, CRP, which is one of the most commonly used biomarkers of infection and inflammation [26], significantly decreased after treatment with rTM not only in survivors and but also in nonsurvivors. These results suggested that rTM, in addition to antibiotics, played important roles in ameliorating inflammation.

In the present study, severity of sepsis indicated by APACHE II and SOFA scores before treatment with rTM was more significantly associated with mortality, compared with severity of coagulopathy indicated by the DIC score. Among coagulation-related variables examined before rTM, only PT-INR values were only marginally associated with mortality. After treatment with rTM, however, all coagulation-related variables improved significantly in survivors, but not in nonsurvivors. These results suggested that not only favorable responses of sepsis to therapies with antibiotics and rTM, but also favorable responses of DIC to them were crucial for improving the patients' outcome.

Since the background of sepsis is highly heterogeneous, it is impossible to find one treatment to fit all situations. Therefore, clinicians have to select appropriate patients who are well suited to any supportive therapy including rTM. Using transcriptomics, a previous study classified bacterial sepsis into three subtypes, including "Inflammopathic", "Adaptive", and "Coagulopathic" subtypes, among which the "Coagulopathic" subtype with more significant coagulopathy was associated with higher mortality, suggesting that the early development of hypercoagulability in sepsis is associated with higher mortality [27]. Reportedly, rTM could effectively suppress hypercoagulability indicated by increased levels of prothrombin fragment 1.2 and thrombin-antithrombin complex, compared with a placebo [28]. Further, rTM-treated patients who had higher baseline levels of prothrombin fragment 1.2 and thrombinantithrombin complex had lower mortality than placebo-treated patients, suggesting that rTM is effective in reducing mortality in patients with hypercoagulability [17]. On the other hand, because the anticoagulant effects of rTM are dependent on thrombin activity in circulation, the ability of rTM to activate protein $\mathrm{C}$ and thus, the anticoagulant effect of rTM are expected to be reduced after thrombin depletion $[5,6]$. The present study showed that patients with milder sepsis and milder coagulopathy before treatment with rTM were significantly more likely to attain DIC resolution, and patients who attained DIC resolution were associated with significantly less mortality. In this regard, diagnosing DIC in 
early phases of sepsis and starting treatment of DIC with rTM before depletion of coagulation factors might be crucial for inducing DIC resolution and reducing mortality in patients with sepsis-induced DIC.

This study had several limitations. First, only a small number of patients were included because of the study conducted at a single institute. Second, the data were retrospectively collected. Third, there was no control group. Because rTM therapy has been recognized as a standard treatment for sepsis-induced DIC in Japan, ethics consideration prevented us from allocating patients to a group without rTM. Alternatively, we compared coagulation- and inflammation-related variables before and after treatment with rTM. Further, we compared these variables between survivors and nonsurvivors, and between patients attaining and not attaining DIC resolution. Clearly, further studies are required to verify the efficacy of rTM in patients with sepsis-induced DIC.

\section{Conclusions}

In the present study, the DIC score significantly reduced after rTM administration in the total cohort, and not only in survivors but also in nonsurvivors. After treatment with rTM, the platelet count, PT-INR values, and FDP levels significantly improved in survivors, but not in nonsurvivors. The platelet count before treatment with rTM was significantly higher while PT-INR values and the DIC score before treatment were significantly lower in patients attaining than not attaining DIC resolution. Patients attaining DIC resolution was associated with lower mortality. Therefore, rTM may play an important role in improving DIC in septic patients, especially when treatment with rTM is initiated in the early phase of DIC, although further studies are needed to verify its efficacy in patients with sepsis-induced DIC.

\section{Abbreviations}

APACHE II: Acute Physiology and Chronic Health Evaluation II; CRP: C-reactive protein; DIC: Disseminated intravascular coagulation; FDP: Fibrin/fibrinogen degradation products; HMGB1: High-mobility group B1 DNA-binding protein; IQR: Interquartile range; JAAM: Japanese Association for Acute Medicine; PT-INR: Prothrombin international normalized ratio; rTM: Recombinant human soluble thrombomodulin; SOFA: Sequential Organ Failure Assessment; TM: Thrombomodulin

\section{Declarations}

\section{Ethics approval and consent to participate}

The study protocol was approved by the Ethics Committee of Juntendo University Hospital (approval number 19-045), with a waiver of patients' written informed consent.

\section{Consent for publication}

Not applicable 
Availability of data and materials

The datasets used and/or analyzed during the current study are available from the corresponding author on reasonable request.

\section{Competing interests}

The authors declare that they have no competing interests.

\section{Funding}

None

\section{Authors' contributions}

$\mathrm{CM}$ designed the protocol and prepared the data listing. $\mathrm{CM}$ and IK performed data collection. CM and DS analyzed the data and performed the statistical analysis. CM drafted the manuscript. $\mathrm{MH}$ edited the manuscript and supervised the study. All authors read and approved the final manuscript.

\section{Acknowledgements}

None

\section{References}

1. Levi M, Cate H. Disseminated intravascular coagulation. N Engl J Med. 1999;341:586-92.

2. Zeerleder S, Hack CE, Wuillemin WA. Disseminated intravascular coagulation in sepsis. Chest. 2005;128:2864-75.

3. Okamoto K, Tamura T, Sawatsubashi Y. Sepsis and disseminated intravascular coagulation. J Intensive Care. 2016;4:23.

4. Levi M, van der Poll T. Inflammation and coagulation. Crit Care Med. 2010;38[Suppl.]:S26-S34.

5. Ikezoe T. Thrombomodulin/activated protein C system in septic disseminated intravascular coagulation. J Intensive Care. 2015;3:1.

6. Loghmani H, Conway EM. Exploring traditional and nontraditional roles for thrombomodulin. Blood. 2018;132:148-58.

7. Okamoto T, Tanigami H, Suzuki K, Shimaoka M. Thrombomodulin: a bifunctional modulator of inflammation and coagulation in sepsis. Crit Care Res Pract. 2012;2012:614545.

8. Ito T, Maruyama I. Thrombomodulin: protectorate God of the vasculature in thrombosis and inflammation. J Thromb Haemost. 2011 Jul;9 Suppl.1:168 - 73.

9. Van de Wouwer M, Plaisance S, De Vriese A, Walkens E, Collen D, Persson J, et al. The lectin-like domain of thrombomodulin interferes with complement activation and protect against arthritis. $J$ Thromb Haemost. 2006;4:1813-24. 
10. Abeyama K, Stern DM, Ito Y, Kawahara K, Yoshimoto Y, Tanaka M, et al. The N-terminal domain of thrombomodulin sequesters high-mobility group-B1 protein, a novel anti-inflammatory mechanism. J Clin Invest. 2005;115:1267-74.

11. Nagato $M$, Okamoto $K$, Abe $Y$, Higure A, Yamaguchi K. Recombinant human soluble thrombomodulin decreases the plasma high-mobility group box-1protein levels, whereas improving the acute liver injury and survival rates in experimental endotoxemia. Crit Care Med. 2009;37:2181-6.

12. Wang H, Yang H, Czura CJ, Sama AE, Tracey KJ. HMGB1 as a late mediator of lethal systemic inflammation. Am J Respir Crit Care Med. 2001;164:1768-73.

13. Saito H, Maruyama I, Shimazaki S, Yamamoto Y, Aikawa N, Ohno R, et al. Efficacy and safety of recombinant human soluble thrombomodulin (ART-123) in disseminated intravascular coagulation: results of a phase III, randomized, double-blind clinical trial. J Thromb Haemost. 2007;5:31-41.

14. Yamakawa K, Ogura H, Fujimi S, Morikawa M, Ogawa M, Mohri T, et al. Recombinant human soluble thrombomodulin in sepsis-induced disseminated intravascular coagulation: a multicenter propensity score analysis. Intensive Care Med. 2013;39:644-52.

15. Hayakawa M, Yamakawa K, Saito S, Uchhino S, Kudo D, lizuka Y, et al. Recombinant human soluble thrombomodulin and mortality in sepsis-induced disseminated intravascular coagulation. A multicentre retrospective study. Thrombo Haemost. 2016;115:1157-66.

16. Vincent JL, Francois B, Zabolotskikh I, Daga MK, Lascarrou J-B, Kirov MY, et al. Effect of a recombinant human soluble thrombomodulin on mortality in patients with sepsis-associated coagulopathy: the SCARLET randomized clinical trial. JAMA. 2019;321:1993-2002.

17. Levi M, Vincent JL, Tanaka K, Eng M, Radford AH, Kayanoki T, et al. Effect of a recombinant human soluble thrombomodulin on baseline coagulation biomarker levels and mortality outcome in patients with sepsis-associated coagulopathy. Crit Care Med. 2020;48:1140-7.

18. Singer MS, Deutschman CS, Seymour CW, Shankar-Haru M, Annane D, Bauer M, et al. The third international consensus definitions for sepsis and septic shock (Sepsis-3). JAMA. 2016;315:801-10.

19. Gando S, Iba T, Eguchi Y, Ohtomo Y, Okamoto K, Koseki K, et al. A multicenter, prospective validation of disseminated intravascular coagulation diagnostic criteria for critically ill patients: Comparing current criteria. Crit Care Med. 2006;34:625-31.

20. Knaus WA, Draper EA, Wagner DP, Zimmerman JE. APACHE II: a severity of disease classification system. Crit Care Med. 1985;13:818-29.

21. Vincent JL, de Mendonça A, Cantraine F, Moreno R, Takala J, Suter PM, et al. Use of the SOFA score to assess the incidence of organ dysfunction/failure in intensive care units: results of a multicenter, prospective study. Working group on "sepsis-related problems" of the European Society of Intensive Care Medicine. Crit Care Med. 1998;26:1793-800.

22. Eguchi $Y$, Gando S, Ishikura H, Saitoh D, Mimuro J. Takahashi H, et al. Post-marketing surveillance data of thrombomodulin alfa: sub-analysis in patients with sepsis-induced disseminated intravascular coagulation. J Intensive Care. 2014;2:30. 
23. Faust SN, Levin M, Harrison OB, Goldin RD, Lockhart MS, Kondaveeti S, et al. Dysfunction of endothelial protein C activation in severe meningococcal sepsis. N Engl J Med. 2001;345(6):408-16.

24. Arishima T, Ito T, Yasuda T, Yashima N, Furubeppu H, Kamikokuryo $C$, et al. Circulating activated protein $\mathrm{C}$ levels are not increased in septic patients treated with recombinant human soluble thrombomodulin. Thromb J. 2018;16:24.

25. Shi CS, Shi GY, Hsiao HM, Kao YC, Kuo KL, Ma CY, et al. Lectin-like domain of thrombomodulin binds to its specific ligand lewis $Y$ antigen and neutralizes lipopolysaccharide-induced inflammatory response. Blood. 2008;112:3661-70.

26. Lelubre C, Anselin S, Boudjeltia KZ, Biston P, Piagnerelli M. Interpretation of C-reactive protein concentrations in critically ill patients. Biomed Res Int. 2013;2013:124021.

27. Sweeney TE, Azad TJ, Donato M, Haynee WA, Perumal TM, Henao R, et al. Unsuperanalysis of transcriptomics in bacterial sepsis multiple datasets reveals three robust clusters. Crit Care Med. 2018;46:915-25.

28. Hoppensteadt D, Tsuruta K, Cunanan J, Hirman J, Kaul I, Osawa Y, et al. Thrombin generation mediators and markers in sepsis-associated coagulopathy and their modulation by recombinant thrombomodulin. Clin Appl Thromb Hemost. 2014 Mar;20:129-35.

\section{Tables}

Table 1 Baseline characteristics in patients with sepsis-induced DIC 


\begin{tabular}{|c|c|c|c|c|c|}
\hline Variables & $\begin{array}{l}\text { Total cohort } \\
(\mathrm{n}=123)\end{array}$ & $\begin{array}{l}\text { Survivors } \\
(\mathrm{n}=98)\end{array}$ & & $\begin{array}{l}\text { Nonsurvivors } \\
(\mathrm{n}=25)\end{array}$ & $P$ value \\
\hline Age (years) & $70[60-78]$ & $70[58-78]$ & & $75[62-79]$ & 0.1736 \\
\hline Male (n $[\%])$ & $66(53.6)$ & $51(52.0)$ & & $15(60.0)$ & 0.4762 \\
\hline APACHE II score & $18[14-24]$ & $17.5[14-22]$ & & $22[16-29]$ & 0.0060 \\
\hline SOFA score & $8[6-11]$ & $8[5-11]$ & & $10[8-13]$ & 0.0018 \\
\hline Surgical interventions & $26(21.1 \%)$ & $21(21.4 \%)$ & & $5(20.0 \%)$ & 0.8759 \\
\hline Sites of infection & & & & & 0.9486 \\
\hline Abdomen & $55(44.7)$ & $44(44.8)$ & 1 & $11(44.0)$ & \\
\hline Urinary tract & $26(21.1)$ & $22(22.4)$ & & $4(16.0)$ & \\
\hline Lung & $19(15.5)$ & $14(14.2)$ & & $5(20.0)$ & \\
\hline Blood & $10(8.1)$ & $8(8.1)$ & & $2(8.0)$ & \\
\hline Skin and soft tissue & $7(5.6)$ & $5(5.1)$ & & $2(8.0)$ & \\
\hline Unknown & $6(4.8)$ & $5(5.1)$ & & $1(4.0)$ & \\
\hline Microorganisms $^{a}$ & & & & & 0.7514 \\
\hline Gram negative & $51(41.4)$ & $41(41.8)$ & & $10(40.0)$ & \\
\hline Gram positive & $23(18.6)$ & $19(19.4)$ & & $4(16.0)$ & \\
\hline Mixed & $20(16.2)$ & $14(14.3)$ & & $6(24.0)$ & \\
\hline No growth & $24(19.5)$ & 19 (19.4) & & $5(20.0)$ & \\
\hline Antibiotic-resistant bacteria $^{b}$ & $8(6.5)$ & $7(7.1)$ & & $1(4.0)$ & 0.5695 \\
\hline Bacteremia $^{c}$ & $68(55.2)$ & $51(52.0)$ & & $17(68.0)$ & 0.1519 \\
\hline
\end{tabular}

The qualitative data are shown as number (percentage) and the quantitative data are shown as medians [interquartile range]. APACHE, acute physiology and chronic health evaluation; DIC, disseminated intravascular coagulation; SOFA, sequential organ failure assessment.

" Microorganisms were determined by culture from blood, sputum, urine, fluid, or abscess.

${ }^{b}$ Antibiotic-resistant bacteria included extended-spectrum beta-lactamase Klebsiella pneumoniae and Escherichia coli, carbapenem-resistant Stenotrophomonas maltophilia, and vancomycin-resistant Enterococcus faecium detected in cultures of seven patients in survivors, and carbapenemresistant Stenotrophomonas maltophilia detected in the culture of one patient in non-survivors.

'Bacteremia is defined as bacteria detected in the bloodstream documented in microbiology reports.

$P$ value. Survivors vs. Nonsurvivors

Table 2 DIC score, platelet count, coagulation biomarkers, and CRP level before and after rTM administration in patients with sepsis-induced DIC

\begin{tabular}{llllll}
\hline Variables & & $\begin{array}{l}\text { Total cohort } \\
(\mathrm{n}=123)\end{array}$ & $\begin{array}{l}\text { Survivors } \\
(\mathrm{n}=98)\end{array}$ & $\begin{array}{l}\text { Nonsurvivors } \\
(\mathrm{n}=25)\end{array}$ & $P$ value \\
\hline DIC score & Before & $6[5-6]$ & $6[5-6]$ & $6[5-8]$ & 0.6463 \\
& After & $2[1-4]^{* * *}$ & $1[1-3]^{* * *}$ & $4[3-5]^{* *}$ & $<0.0001$ \\
Platelet count & Before & $78[49.5-101.5]$ & $81[50.3-107.8]$ & $56[36-80]$ & 0.0742 \\
$\left(\times 10^{\circ} / \mathrm{L}\right)$ & After & $110[79.0-193.5]^{* * *}$ & $129[84.8-209.3]^{* * *}$ & $37[25-85]$ & $<0.0001$ \\
PT-INR & Before & $1.47[1.28-1.75]^{* *}$ & $1.42[1.27-1.62]$ & $1.70[1.31-2.07]$ & 0.0395 \\
& After & $1.18[1.11-1.37]^{* * *}$ & $1.16[1.07-1.27]^{* * *}$ & $1.65[1.34-2.09]$ & $<0.0001$ \\
FDP & Before & $30.0[14.35-49.4]^{*}$ & $27.55[14.23-49.8]$ & $32.9[16.82-44.6]$ & 0.7391 \\
$(\mu \mathrm{g} / \mathrm{mL})$ & After & $11.27[6.45-18.8]^{* * *}$ & $9.45[6.00-16.73]^{* * *}$ & $20.11[11.98-37.8]$ & 0.0038 \\
$\mathrm{CRP}$ & Before & $17.76[10.78-23.76]$ & $17.2[10.47-23.40]$ & $21.60[13.30-24.30]$ & 0.2823 \\
$(\mathrm{mg} / \mathrm{dL})$ & After & $6.22[3.00-12.33]^{* * *}$ & $5.2[2.30-10.87]^{* * *}$ & $12.29[8.76-17.22]^{* *}$ & $<0.0001$ \\
\hline
\end{tabular}

Data are shown as median [interquartile range]. CRP, C-reactive protein; DIC, disseminated intravascular coagulation; FDP, fibrin/fibrinogen degradation products; PT-INR, prothrombin international normalized ratio; rTM, recombinant human soluble thrombomodulin.

$* * p<0.001, * * * p<0.0001$ vs. before rTM, $P$ value, Survivors vs. Nonsurvivors

Table 3 Comparison of various variables before rTM administration between patients attaining DIC resolution and those not attaining DIC resolution 


\begin{tabular}{llll}
\hline DIC resolution after rTM & $\begin{array}{l}\text { DIC resolution } \\
(\mathrm{n}=87)\end{array}$ & $\begin{array}{l}\text { no DIC resolution } \\
(\mathrm{n}=36)\end{array}$ & $P$ value \\
\hline Age, years & $71[59-78]$ & $70[62-79]$ & 0.9733 \\
Male, $\mathrm{n}(\%)$ & $48(55.1)$ & $18(50.0)$ & 0.6006 \\
APACHE II score & $18[14-22]$ & $21[17-28]$ & 0.0071 \\
SOFA score & $8[5-11]$ & $10[8-14]$ & 0.0002 \\
DIC score & $5[4-6]$ & $6[6-8]$ & $<0.0001$ \\
Platelet count $\left(\times 10^{9} / \mathrm{L}\right)$ & $85[55-118]$ & $53[30-80]$ & $<0.0001$ \\
PT-INR & $1.42[1.26-1.59]$ & $1.71[1.38-2.27]$ & 0.0029 \\
FDP $(\mu \mathrm{g} / \mathrm{mL})$ & $26.0[12.8-51.9]$ & $32.7[23.0-42.1]$ & 0.3155 \\
CRP $(\mathrm{mg} / \mathrm{dL})$ & $17.3[11.2-23.6]$ & $18.8[10.4-23.9]$ & 0.9820 \\
\hline
\end{tabular}

Data are shown as median [interquartile range]. APACHE, acute physiology and chronic health evaluation; DIC, disseminated intravascular coagulation; FDP, fibrin/fibrinogen degradation products; PT-INR, prothrombin international normalized ratio; rTM, recombinant human soluble thrombomodulin; SOFA, sequential organ failure assessment.

$P$ value, DIC resolution vs. no DIC resolution 